\section{P57 UNRECOGNISED SCURVY WITH HOME PARENTERAL} NUTRITION

Rulla Al-Araji, Sue Protheroe. Birmingham Children's Hospital

\subsection{6/flgastro-2021-bspghan.66}

Introduction The classical dermatological signs of vitamin C deficiency are now rare. The diagnosis of Scurvy was initially unrecognised in a 16-year-old boy.

Aim To describe a challenging case of vitamin C deficiency. Subject and Method A 16-year-old boy with intestinal failure due to short bowel syndrome. He had been on home parenteral nutrition (PN) for 14 years. He had restricted dietary intake due to intestinal rapid transit.

He presented with 4 weeks history of non-pruritic rash on his legs and 2 weeks of bruising with ankle and knee pain.

On examination, he was systemically well. There were numerous peri-follicular petechiae distributed symmetrically over his lower legs with a few scattered lesions on the arms and trunk. (see figures 1,2).

Initial investigations did not reveal blood dyscrasia, infection, or autoimmune disorder. Vitamin A, D, E, B1, B12, folate, Zinc, Copper, Selenium, and Manganese were within normal limits.

Rheumatology and dermatology opinions were sought; diagnosis of Scurvy was suspected. Skin Biopsy showed perifolliculitis with erythrocyte extravasation suggestive of Scurvy.

Four days after starting vitamin C supplement, symptoms completely resolved. Subsequently, plasma vitamin C level came back low at $1.6 \mu \mathrm{mol} / \mathrm{L} \quad(<11 \mu \mathrm{mol} / \mathrm{L}$ indicates deficiency).

Immediate contact was made with the compounding company and an enquiry revealed inadvertent use of a uni-layer bag for his home PN, allowing accelerated degradation despite addition of the appropriate amount at manufacture.

Summary and discussion $\mathrm{PN}$ is a lifesaving treatment for patients who cannot be adequately nourished by other feeding routes. Despite this, nutritional deficiencies still occur, warranting close nutritional monitoring.

Analysis of ascorbic acid in the uni-layer bag showed degradation by $50 \%$ every 24 hours due to oxidation and by 14 days virtually none was detected. Our patient used bags stored for up to 14 days. In a multi-layer bag, 50\% degradation occurs over 14 days, but the amount remaining in the bag was above the recommended daily allowance of

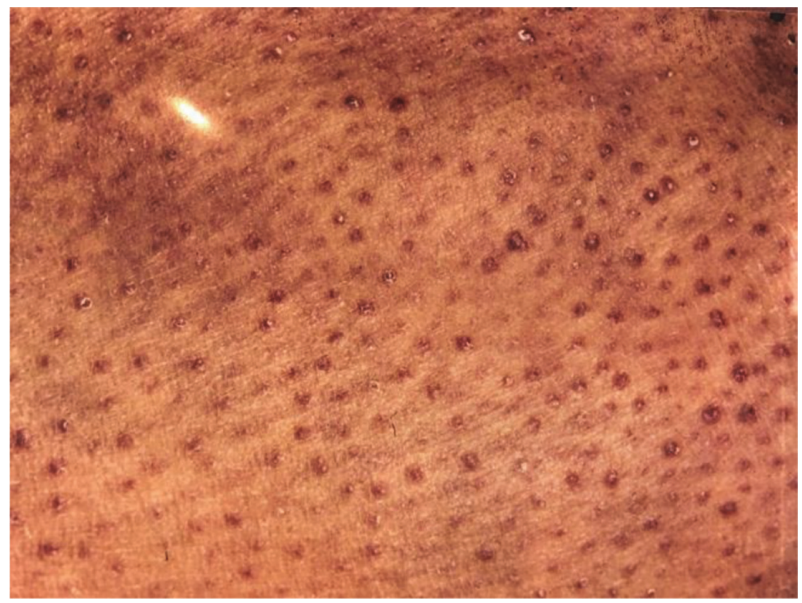

Abstract P57 Figure 1 I - Perifollicular Petechiae

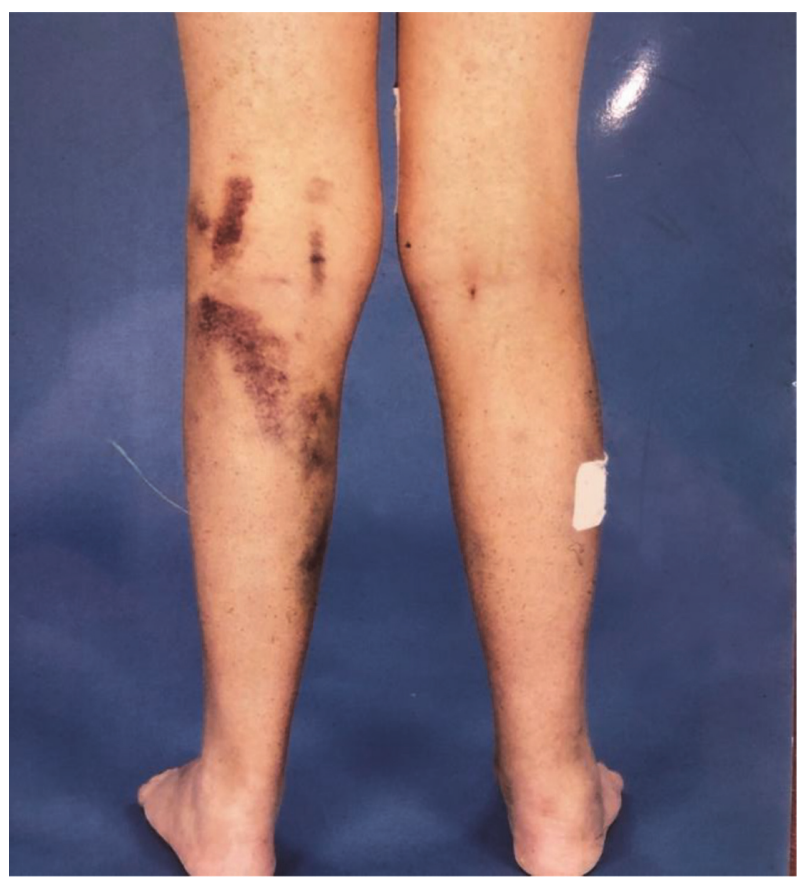

Abstract P57 Figure 2 II Bruising and petechiae

$100 \mathrm{mg} /$ day. Rate of vitamin C decay depends on the constituents of $\mathrm{PN}$, storage environment and the bag containing it $(1,2)$.

Conclusion This case is a reminder of the Scurvy's clinical presentation. Diagnosis was initially challenging; however, starting treatment on clinical suspicion while awaiting lab results relieved the symptoms and substantiated the diagnosis.

Scurvy is preventable and may occur with well monitored PN support as well as in fully enterally fed children (3). We advise to ensure home PN bags are multi-layered and practice stock rotation not only when nearing the expiry date.

Note: Consent was obtained to publish the photos.

\section{REFERENCES}

1. Gibbons E, Allwood MC, Neal T, Hardy G. Degradation of dehydroascorbic acid in parenteral nutrition mixtures. J Pharm Biomed Anal.2001;25(3-4):605-611

2. Gorman $S R$, Armstrong $G$, Allen $K R$, etal. Scarcity in the midst of plenty: enteral tube feeding complicated by Scurvy. I Pediatric Gastroenterology Nutr 2002; 35:93-5

\section{P58 UPTAKE OF NO- BIOPSY APPROACH IN DIAGNOSING PAEDIATRIC COELIAC DISEASE IN A REGIONAL TERTIARY CENTRE}

${ }^{1}$ Predheeba Muthusamy, ${ }^{2}$ Victoria Thomas, ${ }^{3}$ Maureen Lawson. ${ }^{1}$ James Cook University Hospital, Middlesbrough; 'The Great North Children's Hospital, Newcastle Upon Tyne; ${ }^{3}$ Manchester University NHS Foundation Trust, Manchester

\subsection{6/flgastro-2021-bspghan.67}

Background Coeliac disease (CD) is an immune mediated systemic disorder strongly associated with HLA DQ2 and DQ8 haplotypes. $^{2}$

In 2012 The European Society for Paediatric Gastroenterology Hepatology and Nutrition (ESPGHAN) recommended serology based No-Biopsy Approach to the diagnosis of CD if the following criteria are met $^{1}$ : 